\section{Ultra-broad bandwidth and low-loss GCPW-MS transitions on low-k substrates}

M. El-Gibari, D. Averty, C. Lupi, M. Brunet, H. Li and S. Toutain

A study of back-to-back grounded coplanar waveguide-microstrip (GCPW-MS) transitions is presented. By using low-permittivity substrates, both the attenuation and the bandwidth can be improved. An ultra-broad bandwidth up to $77 \mathrm{GHz}$ could be obtained with the $\mathrm{BCB}$ polymer as substrate in thin film on low-resistivity silicon wafers.

Introduction: With the development of MOS transistors of submicron gate length, their cutoff frequency can attain up to $200 \mathrm{GHz}$ [1]. There has hence been a renewed interest in silicon technology for applications in the millimetre waveband in recent years. But realisation of millimetre-wave circuits of high performance requires also good passive elements, such as low-loss and broad-bandwidth transmission lines and transitions to connect active components. The low resistivity of silicon wafers (usually less than $10 \Omega \mathrm{cm}$ ) commonly used in MOS technology is a major handicap because it results in high dielectric losses [2, 3]. Many methods have been studied to improve the poor RF performance of low-resistivity silicon (LRS) substrate. The most cost-effective technique consists in depositing a layer of high-resistivity substrate (e.g. polymer) on LRS wafers. A metal layer deposited between the LRS wafer and the substrate can prevent electromagnetic waves from being propagated into the loss-inducing LRS wafers, so loss is greatly reduced and passive components can be built with relatively high $Q$-factor $[3,4]$. According to the chosen type of technology, a portion of parallel plate waveguides can then create the possibility of parasitic resonances, which reduce the bandwidth of the circuits [5-7]. Via-holes could eliminate some resonance peaks if they are appropriately located but this operation takes time and is expensive to do [6]. Instead of using via-holes to eliminate parasitic resonance, we show that, with thin low- $k$ substrate on LRS wafers, one can realise cost-effective, low-loss and ultra-broad bandwidth GCPW-MS transitions by increasing the parasitic resonance frequencies. Via-free GCPW-MS transitions can considerably facilitate the characterisation with the help of CPW probes of components driven by MS transmission lines, as is the case for electro-optic modulators based on polymer [8].

Topology of studied transitions and measurement conditions: The backto-back GCPW-MS-GCPW transitions studied in this Letter are shown in Fig. 1. The backside conductor used as ground plane for the microstrip line is not patterned. The backside ground of the microstrip and the coplanar ground are electrically short-circuited above a certain frequency owing to electromagnetic coupling between them. We measured the $S$-parameters of the realised transitions with a network analyser Agilent E8364B, the bandwidth of which covers the frequency range from $10 \mathrm{MHz}$ to $50 \mathrm{GHz}$, using probes SuSSMicrotec 50A3N500GSG (bandwidth $40 \mathrm{GHz}$, pitch $500 \mu \mathrm{m}$ and pad $30 \mu \mathrm{m}$ ) and a calibration substrate SussMicrotec CSR-4.

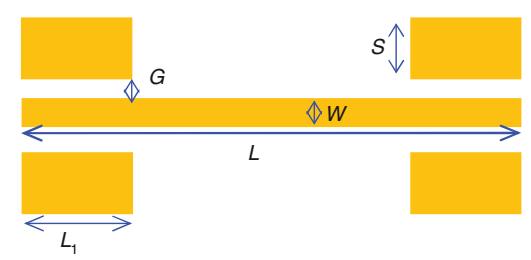

Fig. 1 Structure of studied back-to-back GCPW-MS-GCPW transitions

Simulation and measurement results with $254 \mu$ m-thick substrates: The measured transitions are built with standard commercial substrates of three different permittivities for comparison: $\mathrm{RO} 3006\left(\varepsilon_{r}=3, \tan \delta=\right.$ 0.0013 at $10 \mathrm{GHz}), \mathrm{RO} 3006\left(\varepsilon_{r}=6.15, \tan \delta=0.0025\right)$ and RO3010 $\left(\varepsilon_{r}=10.2, \tan \delta=0.0035\right)$. They are metallised on both sides with $17 \mu \mathrm{m}$ copper. The dimensions of the transitions are determined in order to have a $50 \Omega$ characteristic impedance for both the MS and the GCPW sections. For example, with the substrate RO3003, the width $W$ of the centre strip is $615 \mu \mathrm{m}$ and the coplanar gap $G$ is $185 \mu \mathrm{m}$. The length $L$ of the microstrip line is $1 \mathrm{~cm}$. The CPW pads are $S=1 \mathrm{~mm}$ wide and $L_{1}=1 \mathrm{~mm}$ long. All simulation results were obtained with commercial electromagnetic analysis software for 3D microwave structures (HFSS) by taking into account the CPW probe dimensions used in the measurement. The calculated and the corresponding measured $S$-parameters are presented on the left and the right of Fig. 2, respectively. All the experimental and simulation results are in perfect agreement.
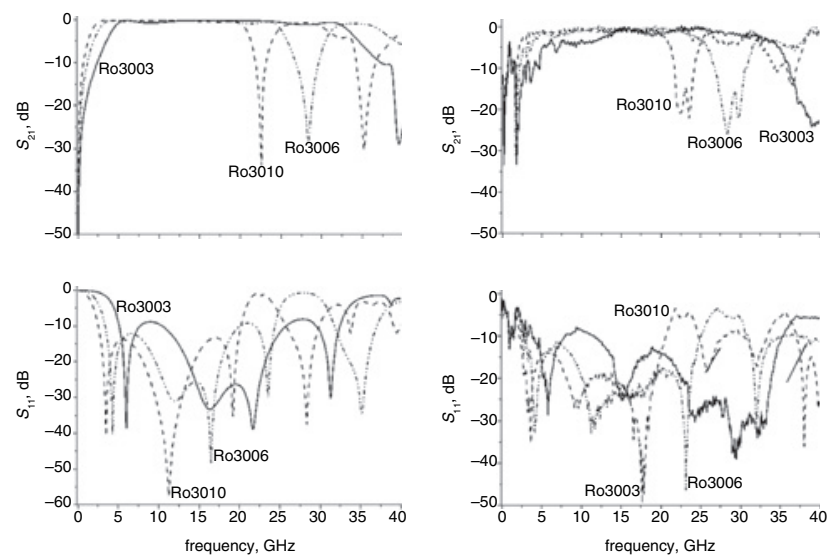

Fig. 2 Calculated (left) and measured (right) S-parameters of back-to-back GCPW-MS-GCPW transition on $254 \mu \mathrm{m}$ substrates with different permittivities

The high cutoff frequencies of the transitions due to the first parasitic resonance peak are, respectively, 20.6, 25 and $33.4 \mathrm{GHz}$ for substrates of permittivities of $10.2,6.15$ and 3 . Owing to the electromagnetic coupling between the backside conductor and the coplanar ground strips, the low cutoff frequency increases with decreasing permittivity, respectively, to $1.7,2.4$ and $4 \mathrm{GHz}$.

Discussion and ultra-broad bandwidth transition on thin BCB substrate on LRS: Low effective permittivity obtained by micromachining substrates allows us to obtain low-dispersive waveguides (quasi TEM approximation) and to realise CPW transmission lines with very high bandwidth without excessive dispersion and loss [9]. The main reason for improvement of the high cutoff frequency of GCPW-MS-GCPW transitions is that the wavelength is bigger in low- $k$ substrate for a given frequency, so the parasitic resonance between the backside conductor and the coplanar ground strips takes place at higher frequencies. In addition, as for low effective permittivities a large part of the energy is propagating in air medium, so losses can be minimised. The parasitic resonance in the GCPW structure has been analysed by Raskin et al. [7]. The electric field propagating in a GCPW line spreads into three modes owing to the backside conductor (see Fig. 3): microstrip mode (MS), coplanar mode (CPW) and coplanar microstrip mode (CPM). The CPM mode is excited between the backside conductor and the coplanar ground strips on the top of the substrate. It does not exist in a CPW structure without a backside conductor. The CPM mode causes the resonance peaks in the $S_{21}$-parameter curve by diverting input electromagnetic energy from the CPW mode. The thickness of the substrate has also an important influence on the CPM mode [10]. One can use a low- $k$ and high resistivity material in thin film for substrate deposited on cheap LRS wafers to push the parasitic resonance to very high frequency, so the high cutoff frequency is increased. In addition the low cutoff frequency can also be improved owing to larger capacitive effect with shorter distance between the backside conductor and the coplanar ground strips.

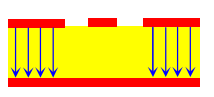

CPM mode

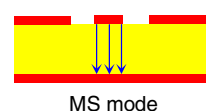

MS mode

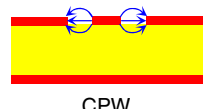

CPW
Fig. 3 Different propagation modes in GCPW structure

The commercial benzocyclobutene polymer (BCB), often used in microelectronic packaging and interconnection applications, is an excellent candidate as a substrate in thin film for GCPW-MS transition on LRS wafers, with its remarkable features: low-permittivity $\left(\varepsilon_{r}=2.65\right)$, low loss tangent $(\tan \delta=0.0025)$, simple and cost-effective to deposit, varying thickness through the spinning-curing process, resistance to 
some chemical agents, etc. [4]. Fig. 4 shows the $S$-parameters obtained by simulation for a $L=1 \mathrm{~cm}$-long back-to-back GCPW-MS transition on $h=8 \mu \mathrm{m}$-thick BCB polymer deposited on a metallised LRS wafer, the metal strips are in $4 \mu \mathrm{m}$ aluminium, the width $W$ of the centre strip is $19 \mu \mathrm{m}$ and the coplanar gap $G$ is $11 \mu \mathrm{m}$. The CPW pads are $S=1 \mathrm{~mm}$ wide and $L_{1}=1 \mathrm{~mm}$ long. The low and the high cutoff frequencies are, respectively, $540 \mathrm{MHz}$ and $77.4 \mathrm{GHz}$.

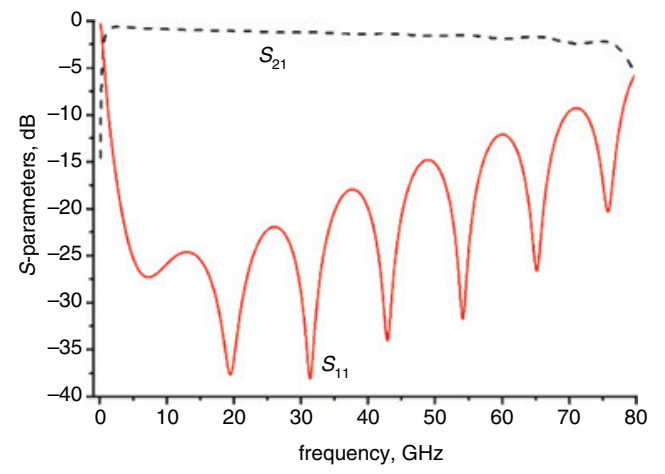

Fig. 4 Calculated S-parameters of back-to-back GCPW-MS-GCPW transition on $8 \mu \mathrm{m} \mathrm{BCB}$ substrate

Conclusions: We have presented in this Letter back-to-back GCPWMS-GCPW via-free transitions on substrates of different permittivities. The low permittivity of the substrate permits pushing into higher frequencies parasitic resonance between the backside conductor and planar ground strips, so the bandwidth of the transitions is enlarged. The bandwidth could also be enlarged by using thin substrates but the requirement of $50 \Omega$ characteristic impedance will limit the centre strip to only $9 \mu \mathrm{m}$ with a $h=8 \mu \mathrm{m}$-thick substrate of permittivity $\varepsilon_{r}=6.15$, which could worsen the adhesion of the metal strip to the substrate, making difficult or even unusable the cost-effective chemical etching of the metal strips owing to the high aspect ratio. In addition, the narrower the centre strip is, the bigger is the ohm effect, and the larger is the negative slope of the $S_{21}$-parameter curve, so band widening by using thin substrates will be limited. Utilisation of low-permittivity and thin substrates permits reducing the negative slope and pushing the parasitic resonance frequency to enlarge the bandwidth of the GCPW-MS transitions, as is shown by the previous simulation results with BCB as substrate on LRS wafers. The bandwidth could go far beyond $60 \mathrm{GHz}$, which opens the perspectives of cost-effective circuits on LRS for $60 \mathrm{GHz}$ wireless communication to undergo rapid expansion.

Acknowledgments: The authors thank Y. Mahé at IREENA for helpful discussion. The work was supported by the Region of Pays de la Loire and the ANR (French National Research Agency) through, respectively, the project Mattador/MILES and the project ModPol.

M. El-Gibari, D. Averty, C. Lupi, M. Brunet, H. Li and S. Toutain (Institut de Recherche en Electrotechnique et Electronique de Nantes Atlantique (IREENA), Université de Nantes, Nantes cedex 3 44322, France)

E-mail: mohammed.el-gibari@univ-nantes.fr

\section{References}

1 O, K.K., Cao, C., Seok, E.-Y., and Sankaran, S.: '100-200 GHz CMOS signal sources and detectors'. IEEE Compound Semiconductor Integrated Circuit Symp., 2007, Portland, USA, October 2007, pp. 1-4

2 Babakhani, A., Xiang, G., Komijani, A., Natarajan, A., and Hajimiri, A.: 'A $77-\mathrm{GHz}$ phased-array transceiver with on-chip antennas in silicon: receiver and antennas', IEEE J. Solid-State Circuits, 2006, 41, pp. 2795-2806

3 Ponchak, G.E.: 'RF transmission lines on silicon substrate'. 29th European Microwave Conf., Munich, Germany, October 1999

4 Wang, J.: 'Polymer-based RF MEMS devices', PhD thesis defended in 2007 at the University of Texas, USA

5 Haydl, W.H.: 'Resonance phenomena and power loss in conductorbacked coplanar structures', IEEE Microw. Guid. Wave Lett., 2000, 10, pp. 514-516

6 Xu, J., Sun, C., Xiong, B., and Luo, Y.: 'Resonance suppression of grounded coplanar waveguide in submount for $40 \mathrm{~Gb} / \mathrm{s}$ optoelectronic modules', J. Infrared Millim. Terahertz Waves, 2009, 30, pp. 103-108

7 Raskin, J.-P., Gauthier, G., Kathei, L.P.B., and Rebeiz, G.M.: 'Mode conversion at GCPW-to-microstrip-line transitions', IEEE Trans. Microw. Theory Tech., 2000, 48, pp. 158-161

8 Michalah, R., Kuo, Y., Nash, F., Szep, A., Caffey, J., Payson, P., Haas, F., Mckeon, B., Cook, P., Brost, G., Luo, J., Jen, A., Dalton, L., and Steier, W.: 'High speed AJL8/APC polymer modulator', IEEE Photonics Technol. Lett., 2006, 18, pp. 1207-1209

9 Cheng, H.-J., Whitaker, J.F., Weller, T.M., and Katehi, L.P.B.: 'Terahertz-bandwidth characteristics of coplanar transmission lines on low permittivity substrates', IEEE Trans. Microw. Theory Tech., 1994, 42, pp. 2399-2406

10 El-Gibari, M., Averty, D., Lupi, C., Li, H.W., and Toutain, S.: 'Ultrawideband GCPW-MS-GCPW driven electrode for low-cost and wide range application electro-optic modulators', Microw. Opt. Technol. Lett., 2010, 52, pp. 1078-1082 